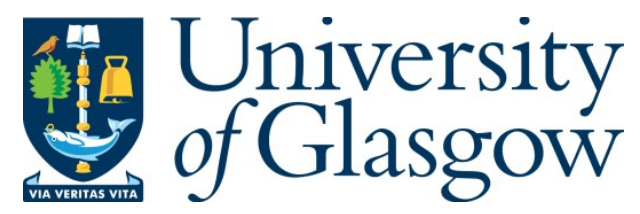

Ewald, C.-O. and Yor, M. (2018) On peacocks and lyrebirds: Australian options, Brownian bridges, and the average of sub-martingales. Mathematical Finance, 28(2), pp. 536-549. (doi:10.1111/mafi.12144).

There may be differences between this version and the published version. You are advised to consult the publisher's version if you wish to cite from it.

This is the peer reviewed version of the following article: Ewald, C.-O. and Yor, M. (2018) On peacocks and lyrebirds: Australian options, Brownian bridges, and the average of sub-martingales. Mathematical Finance, 28(2), pp. 536-549, which has been published in final form at http://dx.doi.org/10.1111/mafi.12144. This article may be used for non-commercial purposes in accordance with Wiley Terms and Conditions for Self-Archiving.

http://eprints.gla.ac.uk/121027/

Deposited on: 12 July 2016

Enlighten - Research publications by members of the University of Glasgow http://eprints.gla.ac.uk 


\title{
On Peacocks and Lyrebirds: Australian Options, Brownian Bridges and the Average of Sub-Martingales
}

\author{
Christian-Oliver Ewald, Marc Yor ${ }^{\dagger}$
}

July 12, 2016

\begin{abstract}
We introduce a class of stochastic processes, which we refer to as lyrebirds. These extend a class of stochastic processes, which have recently been coined peacocks, but are more commonly known as processes which are increasing in the convex order. We show how these processes arise naturally in the context of Asian and Australian Options and consider further applications, such as the arithmetic average of a Brownian bridge and the average of sub-martingales, including the case of Asian and Australian options where the underlying features constant elasticity of variance (CEV) or is of Merton jump diffusion type.
\end{abstract}

Keywords: Stochastic Processes, Convex Order, Asian Options, Brownian Bridges, Random Fields

MSC 2010: 91G20, 60G99

*Adam Smith Business School - Economics, University of Glasgow, Glasgow, G12 8QQ, United Kingdom and School of Mathematics and Statistics, University of Sydney, Sydney, Australia. e-mail: christian.ewald@glasgow.ac.uk

†University Pierre and Marie Curie, Paris, France. Marc Yor passed away on January 9th, 2014. 


\section{Introduction}

In the theory of option pricing, an increased interest in the study of processes which are increasing in the convex order has been observed. This renewed interest originated from the study of certain exotic derivatives, Asian options, and the question about the extent to which their prices depend in a monotonic way on parameters such as time to maturity and volatility, see Carr, Ewald and Xiao (2008) as well as Hirsch et al. (2011). Much earlier, in the late 1960's and 1970's, Strassen (1965), Doob (1968) and Kellerer (1972) obtained key results which characterized such processes as having the same marginals as a martingale. The power of these results now seems to become more and more evident in the context of exotic options pricing.

Definition 1. A stochastic process $\left(X_{t}\right)$ is called increasing in convex order, or peacock, if and only if $\mathbb{E}\left(\left|X_{t}\right|\right)<\infty$ for all $t$ and for every convex function $f(\cdot)$ and $0 \leq s \leq t$ such that both $\mathbb{E}\left(f\left(X_{s}\right)\right)$ and $\mathbb{E}\left(f\left(X_{t}\right)\right)$ are finite we have

$$
\mathbb{E}\left(f\left(X_{s}\right)\right) \leq \mathbb{E}\left(f\left(X_{t}\right)\right)
$$

The name peacock is derived from the French expression for increasing in convex order - Processus Croissant pour l'Ordre Convexe, in short PCOC which is a homophone of the word peacock, see Hirsch et al. (2011). Anecdotal motivation for this name also comes from the fact that the peacock is an Asian bird and the prime example, sometimes also referred to as the guiding peacock, is that of the underlying of an Asian option.

Definition 2. The stochastic process $\left(X_{t}\right)$ is said to have the marginals of a martingale, if and only if there exists a martingale $\left(M_{t}\right)$, such that for each $t \geq 0$

$$
X_{t} \sim M_{t} \text { (equal in distribution). }
$$

Strassen (1965) showed that a discrete time $\mathbb{R}^{d}$-valued stochastic processes is a peacock if and only if it has the marginals of a martingale. Doob 
(1968) proved that a similar result holds for the case of continuous time stochastic processes in a compact metrizable space. Kellerer (1972) then showed that for $\mathbb{R}$-valued processes the martingale can in fact be chosen to be a Markov process. Recent contributions to the peacock literature include, next to the extensive work of Hirsch et al (2011), the articles by Bogso $(2015,2016)$, as well as Ewald and Yor (2015) who discuss applications in the economics of inequality. Further recent applications of peacocks involve Skorohod embeddings, see Källblad et al. (2015) and optimal transport under marginal constraints, see Beiglböck and Juillet (2016).

In this paper, we extend the class of peacocks by restricting the function $f(\cdot)$ in Definition 1 to be monotonic of one type, w.l.o.g. monotonic increasing. This case is often observed in Finance and Economics. As we show, a number of results from the peacock literature can in fact be generalized to this larger class, to which we will refer to as lyrebirds. Lyrebirds will be formally introduced in the next section. In section three, we will show how the Brownian sheet method developed in Baker and Yor (2009) can be generalized to the cases of Asian and Australian options in the Black-Scholes model, where the underlying is allowed to have a drift. This result is the most general of this nature in the context of Asian and Australian options in the Black-Scholes model. We then use the same method in section 4 to show that the average of an exponential Brownian bridge is a lyrebird. This entirely new result has applications in bond and currency options. In section 5, we adopt the previous methodology of Hirsch et al. (2011) to show that a class of functionals involving time averages of sub-martingales are lyrebirds. This provides us with the most general framework for Asian options. Using time reversal techniques, we are able to conclude that under appropriate coefficient conditions the Australian underlying in a Merton type jump diffusion model is a lyrebird. In conclusion, the non-discounted price of an Australian option in this model is increasing with maturity length, which is another important result. Section 6 contains a summary of the main results. 
Some technical computations are included in an appendix.

\section{Lyrebirds and Sub-Martingales}

The use of all convex functions ${ }^{1}$ in Definition 1 restricts the stochastic process $\left(X_{t}\right)$ to have constant expectations. This is often too restrictive for applications. Much of the Finance and Economics literature for example has at its core a potential trade-off between expectation and riskiness. As we demonstrate in the following, a number of stochastic processes occurring naturally in the context of option pricing as well as in econometric applications do not meet the strong peacock condition, but can be considered in a slightly more generalized framework which we refer to as lyrebirds.

Definition 3. The stochastic process $\left(X_{t}\right)$ is called a lyrebird, if $\mathbb{E}\left(\left|X_{t}\right|\right)<$ $\infty$ for all $t$ and $\mathbb{E}\left(h\left(X_{s}\right)\right) \leq \mathbb{E}\left(h\left(X_{t}\right)\right)$ for all $s \leq t$ and increasing convex functions $h(\cdot)$.

The name lyrebird takes its motivation from the following two anecdotes. As much as the guiding peacock is the underlying of an Asian option, the underlying of an Australian option, discussed in more detail in section 3, represents perhaps the most prominent example of a lyrebird. Lyrebirds are native to Australia and while not genetically related to peacocks they are often referred to as peacock-wrens. ${ }^{2}$

In a similar way as peacocks, lyrebirds can be characterized by their marginals too. The following adaptation of the result of Kellerer (1972) holds.

Proposition 1. The process $\left(X_{t}\right)$ is a lyrebird, if and only if $\left(X_{t}\right)$ has the marginals of a sub-martingale.

\footnotetext{
${ }^{1}$ Subject to the integrability condition.

${ }^{2}$ Similar as peacocks male lyrebirds can fan their tails to impress the opposite sex. However, lyrebirds are far better known for their superb ability to mimic natural and artificial sounds.
} 
Proof. Let us first assume that $\left(X_{t}\right)$ has the marginals of a sub-martingale $\left(M_{t}\right)$ and $h(\cdot)$ is an increasing convex function as in Definition 3. It then follows from the Jensen inequality that for $s \leq t$

$$
\begin{aligned}
\mathbb{E}\left(h\left(X_{t}\right)\right) & \left.=\mathbb{E}\left(h\left(M_{t}\right)\right)=\mathbb{E}\left(\mathbb{E}\left(h\left(M_{t}\right) \mid \mathcal{F}_{s}\right)\right)\right) \\
& \geq \mathbb{E}\left(h\left(\mathbb{E}\left(M_{t} \mid \mathcal{F}_{s}\right)\right)\right) \geq \mathbb{E}\left(h\left(M_{s}\right)\right) \\
& =\mathbb{E}\left(h\left(X_{s}\right)\right) .
\end{aligned}
$$

Here, we used in the second line that $\mathbb{E}\left(M_{t} \mid \mathcal{F}_{s}\right) \geq M_{s}$ and that $h(\cdot)$ is increasing.

The reverse implication follows more or less directly from Kellerer's (1972) proof. To see this, note that the class of functions used by Kellerer (1972), i.e., convex functions which are of limiting behavior $\mathcal{O}(\max (x, 0)+1)$ for $x \rightarrow \pm \infty$, slightly differs from the class of functions we use. However in his proof Kellerer (1972) shows that it is sufficient to restrict attention to increasing convex functions of type $h(x)=\max (x, a)$ for all $a \in \mathbb{R}$, which covers our case.

It follows in fact from Kellerer's work that the sub-martingale in Proposition 1 can be chosen to be Markov.

\section{Asian and Australian Options}

It is well known that in the classical Black-Scholes option pricing model, where the risky asset is assumed to follow the geometric Brownian motion

$$
S_{t}=e^{\left(\mu-\frac{1}{2} \sigma^{2}\right) t+\sigma B_{t}},
$$

the price of a European call option increases with both the volatility and time to maturity. However it was unknown until recently, whether prices of options traded on the average price of such an asset, so called Asian options, 
would be increasing in volatility and/or maturity. Carr, Ewald and Xiao (2008) were the first to prove that the price of an Asian option is indeed increasing in the volatility of the asset price and if $\mu=0$, the same is true with respect to time to maturity. A direct consequence of this is that under the assumption of $\mu=0$ the process

$$
X_{t}=\frac{1}{t} \int_{0}^{t} S_{u} d u
$$

is a peacock. Carr, Ewald and Xiao (2008) obtained their result from the maximum principle for parabolic PDEs. Shortly after that, Baker and Yor (2009) generalized the results by Carr, Ewald and Xiao (2008) using Wiener sheet methods. They explicitly constructed a martingale which has the same marginals as the Asian underlying and a large amount of peacock literature developed from then on. The case of an Asian option has been particularly instructive for the construction and study of new peacocks and the example by Carr, Ewald and Xiao (2008) has henceforth been considered as the "guiding" peacock in the literature.

The case $\mu \neq 0$ has somehow been discarded so far. In this case, it is obvious that $\left(X_{t}\right)$ cannot have the marginals of a martingale, as its expectation is not constant. However, as we will show, in the case of $\mu>0$, the process $\left(X_{t}\right)$ is in fact a lyrebird and hence increasing in convex order for increasing convex functions.

Australian options are related to Asian options in the way that an average of the asset price $\left(S_{t}\right)$ is considered for the payoff, but in difference to Asian options, the quotient of the average and the asset price at maturity then determines the payoff, i.e.,

$$
X_{t}=\frac{\frac{1}{t} \int_{0}^{t} S_{u} d u}{S_{t}}
$$


Such options have been traded on the Australian Stock Exchange since 1992, see Handley $(2000,2003)$, from which the name originated. These options are related to Quanto options and Dollar Cost Averaging, see Ewald, Menkens and Ting (2013). Yang, Ewald and Menkens (2011) use Malliavin calculus to derive quasi-explicit hedging strategies for Asian options and their approach can be used to derive similar hedging strategies for Australian options.

In the following we provide an explicit construction of a sub-martingale which has the same marginals as the Australian underlying. For this we adapt the Wiener sheet method introduced by Baker and Yor (2009).

A Wiener sheet is a centered Gaussian process $\left(W_{s, t}\right)$, parametrized by two arguments $s, t \in[0, \infty)$ with the property that

$$
\operatorname{cov}\left(W_{s, t}, W_{u, v}\right)=\min (s, u) \times \min (t, v) .
$$

We denote with $\left(\mathcal{F}_{s, t}\right)$ the filtration generated by the Wiener sheet $\left(W_{s, t}\right)$. It is not difficult to show that with $\left(W_{\nu, t}\right)$ the process $\left(\widetilde{W}_{\nu, t}\right)$ defined via

$$
\widetilde{W}_{\nu, t}:=W_{1, t}-W_{1-\nu, t}
$$

is also a Wiener sheet and as such has the same distribution in the sense of a two parameter stochastic process. This will be useful to unify the argument for the Asian and Australian case. It follows that for all $t \in[0, \infty)$ fixed

$$
\left(B_{\nu t}, \nu \geq 0\right) \sim\left(W_{\nu, t}, \nu \geq 0\right)
$$

have the same law as stochastic processes in $\nu \in[0, \infty)$, where $\left(B_{t}\right)$ denotes the Brownian motion from equation (4). Equation (9) presents the main idea of the Wiener sheet method, through which time averaging over an extending horizon (left hand side) is transformed into averaging over the first parameter 
of a Wiener sheet with fixed horizon (right hand side). In fact equation (9) implies that for general $\mu$ and $\sigma$

$$
\begin{array}{r}
\frac{\frac{1}{t} \int_{0}^{t} d u \exp \left(\sigma B_{u}+\left(\mu-\frac{1}{2} \sigma^{2}\right) u\right)}{\exp \left(\sigma B_{t}+\left(\mu-\frac{1}{2} \sigma^{2}\right) t\right)} \sim \frac{\int_{0}^{1} d \nu \exp \left(\sigma W_{\nu, t}+\left(\mu-\frac{1}{2} \sigma^{2}\right) \nu t\right)}{\exp \left(\sigma W_{1, t}+\left(\mu-\frac{1}{2} \sigma^{2}\right) t\right)} \\
\sim \frac{\int_{0}^{1} d \nu \exp \left(\sigma \widetilde{W}_{\nu, t}+\left(\mu-\frac{1}{2} \sigma^{2}\right) \nu t\right)}{\exp \left(\sigma \widetilde{W}_{1, t}+\left(\mu-\frac{1}{2} \sigma^{2}\right) t\right)} \\
\sim \frac{\int_{0}^{1} d \nu \exp \left(\sigma\left(W_{1, t}-W_{1-\nu, t}\right)+\left(\mu-\frac{1}{2} \sigma^{2}\right) \nu t\right)}{\exp \left(\sigma\left(W_{1, t}-W_{0, t}\right)+\left(\mu-\frac{1}{2} \sigma^{2}\right) t\right)} \\
\sim \int_{0}^{1} d \nu \exp \left(-\sigma W_{1-\nu, t}-\left(\mu-\frac{1}{2} \sigma^{2}\right)(1-\nu) t\right) \\
\sim \int_{0}^{1} d \nu \exp \left(-\sigma W_{\nu, t}-\left(\mu-\frac{1}{2} \sigma^{2}\right) \nu t\right)=: \tilde{M}_{t} .
\end{array}
$$

Here we used (9) and the fact that $\left(\widetilde{W}_{\nu, t}\right)$ is a Wiener sheet in the second and third line as well as that $W_{0, t}=0$. The expression in the last line now is of the same form as the expression obtained in Baker and Yor (2009) for Asian options, with the difference that the exponent appears with a negative sign.

We will now investigate under which conditions $\left(\tilde{M}_{t}\right)$ defined in (10) is a sub-martingale or a super-martingale with respect to the filtration $\left(\mathcal{F}_{\infty, t}\right)$, with $\mathcal{F}_{\infty, t}=\sigma\left(\mathcal{F}_{s, t} \mid s \geq 0\right)$. This analysis is analogous to Baker and Yor (2009). In fact we have

$$
\mathbb{E}\left(\tilde{M}_{t} \mid \mathcal{F}_{\infty, s}\right)=\int_{0}^{1} d \nu \mathbb{E}\left(\exp \left(-\sigma W_{\nu, t}-\left(\mu-\frac{1}{2} \sigma^{2}\right) \nu t\right) \mid \mathcal{F}_{\infty, s}\right)
$$


As $W_{\nu, t}-W_{\nu, s}$ is independent of $\mathcal{F}_{\infty, s}$ we have

$$
\begin{aligned}
\mathbb{E}\left(\exp \left(-\sigma W_{\nu, t}\right) \mid \mathcal{F}_{\infty, s}\right) & =\mathbb{E}\left(\exp \left(-\sigma\left\{W_{\nu, t}-W_{\nu, s}\right\}\right)\right) \exp \left(-\sigma W_{\nu, s}\right) \\
& =\exp \left(\frac{1}{2} \sigma^{2} \nu(t-s)\right) \exp \left(-\sigma W_{\nu, s}\right)
\end{aligned}
$$

where we used that $-\sigma\left\{W_{\nu, t}-W_{\nu, s}\right\}$ is Gaussian with expectation zero and variance $\sigma^{2} \nu(t-s)$. Hence we conclude from (11) that

$$
\begin{aligned}
\mathbb{E}\left(\tilde{M}_{t} \mid \mathcal{F}_{\infty, s}\right)= & \int_{0}^{1} d \nu\left\{\exp \left(-\sigma W_{\nu, s}\right) \times \exp \left(\frac{1}{2} \sigma^{2} \nu(t-s)\right)\right. \\
& \left.\times \exp \left(-\left(\mu-\frac{1}{2} \sigma^{2}\right) \nu t\right)\right\} \\
= & \int_{0}^{1} d \nu \exp \left(-\sigma W_{\nu, s}-\left(\mu-\frac{1}{2} \sigma^{2}\right) \nu s\right) \times \rho(\nu),
\end{aligned}
$$

with

$$
\rho(\nu):=\exp \left(\left(\sigma^{2}-\mu\right) \nu(t-s)\right)
$$

As $s \leq t$ it can be readily observed from (12) and (13) that

$$
\begin{aligned}
& \mu \leq \sigma^{2} \Rightarrow \rho(\nu) \geq 1 \text { for all } \nu \in[0,1] \Rightarrow \mathbb{E}\left(\tilde{M}_{t} \mid \mathcal{F}_{\infty, s}\right) \geq \tilde{M}_{s} \\
& \mu \geq \sigma^{2} \Rightarrow \rho(\nu) \leq 1 \text { for all } \nu \in[0,1] \Rightarrow \mathbb{E}\left(\tilde{M}_{t} \mid \mathcal{F}_{\infty, s}\right) \leq \tilde{M}_{s}
\end{aligned}
$$

This leads to the following proposition which characterizes the Australian case.

Proposition 2. Consider the Australian underlying $\left(X_{t}\right)$ from (6). Then

1. if $\mu<\sigma^{2}$ then $\left(X_{t}\right)$ has the marginals of a sub-martingale and hence is a lyrebird

2. if $\mu>\sigma^{2}$ then $\left(X_{t}\right)$ has the marginals of a super-martingale 
3. if $\mu=\sigma^{2}$ then $\left(X_{t}\right)$ has the marginals of a martingale and hence is a peacock.

In each case, a sub-/super- martingale with the same marginal distribution is explicitly given by (10).

Proof. This follows directly from the discussion above.

These results carry over to the case of Asian options.

Corollary 4. For $\mu>0$, the Asian underlying $X_{t}=\frac{1}{t} \int_{0}^{t} S_{u} d u$ in the BlackScholes model is a lyrebird. In the case of $\mu<0$ it has the same marginals as a super-martingale.

Proof. This follows from Proposition 2 taking account of the general relationship between Australian and Asian options, which has been detailed in Ewald, Menkens and Ting (2013). ${ }^{3}$

Proposition 2 and Corollary 4 imply that the prices of the corresponding Australian and Asian options for $\mu \leq \sigma^{2}$ respectively $\mu \geq 0$ are increasing with maturity length, when the premium is paid at maturity, which means no-discounting (the option is of "pay later" type).

\section{Brownian Bridges and Related Functionals}

A standard Brownian bridge on the interval $[0,1]$ is a Gaussian process $\left(X_{u}\right)$ such that for $0 \leq s \leq u \leq 1$

$$
\operatorname{cov}\left(X_{s}, X_{u}\right)=s(1-u)
$$

\footnotetext{
${ }^{3}$ Alternatively the result can be concluded directly from applying the Baker and Yor (2009) method used to derive Proposition 2 for this particular case.
} 
which starts at $u=0$ with value $X_{0}=0$ and ends at $u=1$ with value $X_{1}=0$. In between, it behaves Brownian motion like, but constrained to the two boundary conditions. A standard Brownian bridge can be constructed as the unique solution of the following stochastic differential equation

$$
d X_{u}=-\frac{1}{1-u} X_{u} d u+d B_{u}
$$

with $\left(B_{u}\right)$ a standard Brownian motion. Note the singularity at $u=1$ in the drift term of this diffusion and that existence and uniqueness of a solution of this SDE do not follow from standard theorems. The theory developed for Brownian bridges has fundamental applications in econometrics as well as the economics of inequality. The reason for this is that a number of limiting distributions, including those corresponding to the order statistics or inequality measures such as the Gini-index, can be expressed in terms of functionals of Brownian bridges. Davidson (2009) provides an excellent source for this. Brownian bridges have also been used in the context of term structure modeling, see Ball and Torous (1983), Cheng (1991) Ajevskis and Vitola (2010).

It is rather obvious that the standard Brownian bridge is neither a peacock nor a lyrebird, as both $X_{0} \equiv 0$ and $X_{1} \equiv 0$ are deterministic, and hence any associated martingale or sub-martingale $\left(M_{t}\right)$ would need to be deterministic on the whole interval $[0,1]$. But then $\left(X_{t}\right)$ would need to be deterministic, which it is not. However $\left(X_{t}\right)$ is a peacock on the interval $\left[0, \frac{1}{2}\right]$ and then turns into something that could be called a backward peacock, i.e., a peacock after time inversion, on $\left[\frac{1}{2}, 1\right]$. This conclusion can be drawn from Hirsch et al. (2011) page 51, who show that for any function $F \in C^{2}$ with compact support the formula

$$
\frac{d}{d u} \mathbb{E}\left(F\left(X_{u}\right)\right)=\left(\frac{1-2 u}{2}\right) \mathbb{E}\left(F^{\prime \prime}\left(X_{u}\right)\right)
$$


holds for all $u \in[0,1]$. In the following, we will look at a more sophisticated functional of the Brownian bridge. In order to do this, we consider exponentials of Brownian bridges on the interval $[0, t]$ for variable $t$ rather than on the interval $[0,1]$. An explicit expression for the exponential of a Brownian bridge on the interval $[0, t]$ is given by

$$
\exp \left(B_{s}-\frac{s}{t} B_{t}\right), s \in[0, t]
$$

see Revuz and Yor (2004) page 35. We now consider the functional which measures the average of such exponentials of Brownian bridges as a function of time $t$, i.e.,

$$
Z_{t}=\frac{1}{t} \int_{0}^{t} \exp \left(B_{s}-\frac{s}{t} B_{t}\right) d s .
$$

Proposition 3. The average of an exponential Brownian bridge $\left(Z_{t}\right)$ as constructed in (16) is a lyrebird.

Proof. We use the methodology based on the Wiener sheet in a similar fashion as in section 3 . Let $\left(W_{\nu, t}\right)$ be a two parameter Wiener sheet as in $(7)$. Then, it follows from (9) in a similar fashion as in the derivation of (10), that

$$
Z_{t} \sim \int_{0}^{1} d \nu \exp \left(W_{\nu, t}-\nu W_{1, t}\right)=: M_{t}
$$

Further, we have that for $t \geq s$

$$
\begin{aligned}
\mathbb{E}\left(M_{t} \mid \mathcal{F}_{\infty, s}\right) & =\int_{0}^{1} d \nu \mathbb{E}\left(\exp \left(W_{\nu, t}-\nu W_{1, t}\right) \mid \mathcal{F}_{\infty, s}\right) \\
& =\int_{0}^{1} d \nu \exp \left(\frac{1}{2} \nu(1-\nu)(t-s)\right) \cdot \exp \left(W_{\nu, s}-\nu W_{1, s}\right) .
\end{aligned}
$$

This follows from the fact that $W_{\nu, t}-W_{\nu, s}$ is independent of $\mathcal{F}_{\infty, s}$ and Lemma A1 in the appendix. As $\nu \in[0,1]$ and $s \leq t$, we have that

$$
\exp \left(\frac{1}{2} \nu(1-\nu)(t-s)\right) \geq 1
$$


which implies that

$$
\mathbb{E}\left(M_{t} \mid \mathcal{F}_{\infty, s}\right) \geq M_{s}
$$

It follows from the proof above that for $s<t$ the inequality in (18) is in fact strict and that $\left(Z_{t}\right)$ is a strict lyrebird, i.e., not a peacock. In the framework of Ball and Torous (1983), an option written on the underlying (16) corresponds to an Asian option on a bond and Proposition 3 implies that the price of such an option is increasing with length of maturity, if the premium is paid at maturity.

\section{Averaging Sub-Martingales}

In this section, we extend certain results obtained for martingales in Hirsch et al. (2011) to the case of sub-martingales, linking to the lyrebird framework. This will in principle lead to many more examples of lyrebirds.

Proposition 4. Let $\left(M_{t}\right)$ be a right-continuous sub-martingale such that

$\mathbb{E}\left(\sup _{0 \leq s \leq t}\left|M_{s}\right|\right)<\infty$ for all $t>0$ and $\alpha:[0, \infty) \rightarrow[0, \infty)$ be an increasing continuous function such that $\alpha(0)=0$. Further, let $g(\cdot)$ and $h(\cdot)$ be two increasing and convex functions. Then the processes $\left(X_{t}\right)$ and $\left(\tilde{X}_{t}\right)$ defined via

$$
\begin{aligned}
& X_{t}:=g\left(\frac{1}{\alpha(t)} \int_{0}^{t} h\left(M_{s}\right) d \alpha(s)\right) \\
& \tilde{X}_{t}:=g\left(\int_{0}^{t}\left(h\left(M_{s}\right)-h\left(M_{0}\right)\right) d \alpha(s)\right)
\end{aligned}
$$

are lyrebirds.

Proof. The proof of these results is strongly linked to the proof of Theorem 1.4 on page 26 in Hirsch et al. (2011). We only point out the differences 
in the following. First, note that as $h(\cdot)$ is increasing and convex, it follows from Jensen's inequality that

$$
\mathbb{E}\left(h\left(M_{t}\right) \mid \mathcal{F}_{s}\right) \geq h\left(\mathbb{E}\left(M_{t} \mid \mathcal{F}_{s}\right)\right) \geq h\left(M_{s}\right)
$$

and hence that $\left(h\left(M_{t}\right)\right)$ is a sub-martingale as well. Therefore we can assume w.l.o.g. that $h(x)$ is the identity function. Further, as the composition of any two convex and increasing functions is also convex and increasing, we can as well assume that $g(\cdot)$ is the identity function too. Now, in order to prove the first point, Hirsch et al. (2011) (in the context of peacocks) use partial integration to represent the process $\left(X_{t}\right)$ as

$$
X_{t}=M_{t}-\frac{1}{\alpha(t)} M_{t}^{\alpha}
$$

with $M_{t}^{\alpha}=\int_{0}^{t} \alpha(s) d M_{s}$ and obtain that

$$
d X_{t}=M_{t}^{\alpha} \frac{d \alpha(t)}{\alpha^{2}(t)}
$$

As $\left(M_{t}\right)$ is no longer a martingale, the proof in Hirsch et al. (2011) now needs to be adjusted in the following way: Note that as the integral of a positive integrand with respect to a sub-martingale, $M_{t}^{\alpha}$ is also a sub-martingale. It then follows from (22) that

$$
\begin{aligned}
\mathbb{E}\left(X_{t} \mid \mathcal{F}_{s}\right) & =X_{s}+\mathbb{E}\left(\int_{s}^{t} M_{u}^{\alpha} \frac{d \alpha(u)}{\alpha^{2}(u)} \mid \mathcal{F}_{s}\right) \\
& \geq X_{s}+M_{s}^{\alpha} \int_{s}^{t} \frac{d \alpha(u)}{\alpha^{2}(u)} \\
& =X_{s}+M_{s}^{\alpha}\left(\frac{1}{\alpha(s)}-\frac{1}{\alpha(t)}\right),
\end{aligned}
$$

where we used in the second line that $M_{u}^{\alpha}$ is a sub-martingale and that $\frac{d \alpha(u)}{\alpha^{2}(u)}$ is deterministic and positive. Now note that in the light of section 2 , in 
difference to Hirsch et al. (2011), we only need to consider increasing convex functions $\psi(x)$ (using the same notation as in Hirsch et al. (2011)). This guarantees that

$$
\mathbb{E}\left(\int_{\epsilon}^{s} \psi^{\prime}\left(X_{u}\right) d M_{u}^{\alpha}\right) \geq 0,
$$

which allows us to complete the proof of the first point exactly as in Hirsch et al. (2011). For the second point, the only relevant issues are that

$$
\int_{s}^{t} \mathbb{E}\left(M_{u} \mid \mathcal{F}_{s}\right) d \alpha(u) \geq(\alpha(t)-\alpha(s)) M_{s}
$$

and because we can restrict ourselves to increasing convex functions $\psi(x)$ that

$$
\mathbb{E}\left(\psi\left(\mathbb{E}\left(\tilde{X}_{t} \mid \mathcal{F}_{s}\right)\right)\right) \geq \mathbb{E}\left(\psi\left(\tilde{X}_{s}+(\alpha(t)-\alpha(s)) M_{s}\right)\right)
$$

at which point the proof can be completed exactly in the same way as in Hirsch et al. (2011).

Proposition 4 leads to many examples of lyrebirds. Let $Q_{s}^{(q, \delta)}$, be a squared Bessel process of dimension $\delta>0$ starting at $q>0$. Then the process

$$
X_{t}^{(\alpha, q, \delta)}=\frac{1}{t^{\alpha+1}} \int_{0}^{t} Q_{s}^{(q, \delta)} s^{\alpha} d s
$$

defined for $t \geq 0$ is a lyrebird according to Proposition 4 , as $Q_{s}^{(q, \delta)}$ is a submartingale. Hirsch et al. (2011) Chapter 4 consider this case for $\delta=0$ only, when $\left(X_{t}\right)$ is in fact a peacock. Further noteworthy in this context is that the inverse of a three dimensional Bessel process $\left(\frac{1}{R_{t}}\right)$ is a local martingale, but not a peacock. Because of its positivity though, it represents a supermartingale, and as such its negative is a lyrebird. The same is true for the average of the process obtained according to Proposition 4.

The above also implies that for any arbitrage free market model with positive interest and asset prices, the Asian underlying $\frac{1}{t} \int_{0}^{t} S_{u} d u$ is a lyrebird, not only for the Black-Scholes model, but in general. This is the most general 
version of the result obtained in Carr, Ewald and Xiao (2008) and includes many practically relevant cases such as the CEV model in which

$$
d S_{t}=\mu S_{t} d t+\sigma S_{t}^{\beta} d B_{t}
$$

with $\beta>0$. For general $\beta$ no closed form expression exist for the solution of the SDE (25), however the cumulative distribution function of $S_{t}$ can be expressed in terms of the chi-square distribution, see Jeanblanc et al. (2009). The evaluation of the terms is however computationally heavy. Chen and Ewald (2014) applied the comonotonicity approach to price Asian options in the CEV framework. However, there had been no results on qualitative issues such as the dependence on time to maturity and volatility of prices of Asian options in the CEV model until now. The framework discussed in this paper helps answering some questions. First, from the above it is clear, that the price of an Asian call option in the CEV model is increasing in the averaging time for general $\mu>0$. If $\mu=0$, the relationship $\sigma d B_{t} \sim d B_{\sigma^{2} t}$ can then be used in combination with time re-parametrization to show that the price of an Asian call in the CEV model is also increasing in the volatility parameter.

Let us now return to consider averages of the Australian type. In comparison to Proposition 4, the next proposition is rather simple to prove as it does not involve the scaling factor $\frac{1}{t}$ in the averages.

Proposition 5. Let $\left(M_{t}\right)$ be a right-continuous positive super-martingale with $\mathbb{E}\left(M_{t}^{-2}\right)<\infty$ for all $t$, then the process

$$
X_{t}:=\frac{\int_{0}^{t} M_{u} d u}{M_{t}}
$$

is a sub-martingale, hence a lyrebird.

Proof. It follows from the Cauchy-Schwartz inequality that $\mathbb{E}\left(\left|X_{t}\right|\right)<\infty$. 
We have that

$$
\begin{aligned}
\mathbb{E}\left(X_{t} \mid \mathcal{F}_{s}\right) & =\mathbb{E}\left(\frac{\int_{0}^{s} M_{u} d u}{M_{t}}+\frac{\int_{s}^{t} M_{u} d u}{M_{t}} \mid \mathcal{F}_{s}\right) \\
& =\left(\int_{0}^{s} M_{u} d u\right) \cdot \mathbb{E}\left(\frac{1}{M_{t}} \mid \mathcal{F}_{s}\right)+\mathbb{E}\left(\frac{\int_{s}^{t} M_{u} d u}{M_{t}} \mid \mathcal{F}_{s}\right) \\
& \geq \frac{\int_{0}^{s} M_{u} d u}{M_{s}}+\mathbb{E}\left(\frac{\int_{s}^{t} M_{u} d u}{M_{t}} \mid \mathcal{F}_{s}\right) \geq X_{s},
\end{aligned}
$$

where we used $\mathbb{E}\left(\frac{1}{M_{t}} \mid \mathcal{F}_{s}\right) \geq \frac{1}{M_{s}}$, noticing that as $\left(M_{t}\right)$ is a positive supermartingale, its inverse is a sub-martingale.

Whether Proposition 5 remains true, at least for a Martingale $\left(M_{t}\right)$, under consideration of the factor $\frac{1}{t}$ which appears in the Australian underlying, is not clear. We have the following Proposition 6, which will be applicable to exponential Lévy processes, as we will show further below.

Proposition 6. Let $\left(M_{t}\right)$ be a strictly positive cadlag $g^{4}$ process such that

1. the time-inversed process $\left(\overleftarrow{X}_{u}^{t}\right)$ with

$$
\overleftarrow{\leftarrow}_{u}^{t}:=\frac{M_{t-u}}{M_{t}} \text { for } u \in[0, t]
$$

is a sub-martingale for a suitable filtration $\left(\overleftarrow{\mathcal{F}}_{u}^{t}\right)$

2. the distribution of $\left(\overleftarrow{\leftarrow}_{u}^{t}\right)$ (as a stochastic process) is consistent in $t$, i.e.,

$$
\left(\overleftarrow{X}_{u}\right) \sim\left(\overleftarrow{H}_{u}\right)
$$

on the interval $[0, \min (s, t)]$.

\footnotetext{
${ }^{4}$ Right continuous with left limits.
} 
Then the Australian process $\left(Z_{t}\right)$ defined via $Z_{t}:=\frac{\frac{1}{t} \int_{0}^{t} M_{u} d u}{M_{t}}$ is a lyrebird.

Proof. Let $\psi(\cdot)$ be a convex and increasing function and $s<t$. Then

$$
\begin{aligned}
\mathbb{E}\left(\psi\left(Z_{t}\right)\right) & =\mathbb{E}\left(\psi\left(\frac{1}{t} \int_{0}^{t} \frac{M_{u}}{M_{t}} d u\right)\right)=\mathbb{E}\left(\psi\left(\frac{1}{t} \int_{0}^{t} \frac{M_{t-u}}{M_{t}} d u\right)\right) \\
& =\mathbb{E}\left(\psi\left(\frac{1}{t} \int_{0}^{t} \overleftarrow{X}_{u} d u\right)\right) \geq \mathbb{E}\left(\psi\left(\frac{1}{s} \int_{0}^{s} \overleftarrow{X}_{u} d u\right)\right) \\
& =\mathbb{E}\left(\psi\left(\frac{1}{s} \int_{0}^{s} \overleftarrow{X}_{u} d u\right)\right)=\mathbb{E}\left(\psi\left(\frac{1}{s} \int_{0}^{s} \frac{M_{u}}{M_{s}} d u\right)\right)=\mathbb{E}\left(\psi\left(Z_{s}\right)\right)
\end{aligned}
$$

To infer the inequality in the second line, we used assumption 1 as well as Proposition 4. To infer the equality in the second line, we used assumption 2 .

The following proposition demonstrates how Proposition 6 can be applied in practice. We consider the Merton (1976) model in which the asset prices follows a jump diffusion of the following type

$$
S_{t}=S_{0} \exp \left(\left(\mu-\lambda \alpha^{*}-\frac{1}{2} \sigma^{2}\right) t+\sigma W_{t}+\sum_{i=1}^{N_{t}} Y_{i}\right)
$$

with $Y_{i} \sim \mathcal{N}\left(\alpha, \delta^{2}\right)$ i.i.d. and $\left(N_{t}\right)$ a Poisson process with intensity $\lambda$ and $\alpha^{*}=$ $\exp \left(\alpha+\frac{\delta^{2}}{2}\right)-1$. The adjustment of the drift term is chosen in consistence with other literature, so as to make the discounted process $\exp (-\mu t) S_{t}$ a martingale. As a consequence $\left(S_{t}\right)$ is also a sub-martingale.

Proposition 7. Under the assumptions of the Merton (1976) jump diffusion model with asset price following (30), if

$$
\sigma^{2} \geq \mu-2 \lambda \cdot\left(\exp \left(\frac{\delta^{2}}{2}\right) \cdot \cosh (\alpha)-1\right)
$$

then the Australian process $\left(\frac{\frac{1}{t} \int_{0}^{t} S_{u} d u}{S_{t}}\right)$ is a lyrebird. 
Proof. Using the notation of Proposition 6, we have that

$$
\stackrel{\leftarrow}{X}_{u}=\frac{S_{t-u}}{S_{t}}=\exp \left(-\left(\mu-\lambda \alpha^{*}-\frac{1}{2} \sigma^{2}\right) u-\sigma\left(W_{t}-W_{t-u}\right)-\sum_{i=N_{t-u}}^{N_{t}} Y_{i}\right)
$$

As is well known, $\overleftarrow{W}_{u}:=W_{t}-W_{t-u}$ defines a Brownian motion for $u \in[0, t]$ and similarly $\overleftarrow{L}_{u}:=-\sum_{i=N_{t-u}}^{N_{t}} Y_{i}$ is a Poisson process for $u \in[0, t]$, with intensity $\lambda$ and jumps $Y_{i} \sim \mathcal{N}\left(-\alpha, \delta^{2}\right)$. Let $\overleftarrow{\alpha}^{*}=\exp \left(-\alpha+\frac{\delta}{2}\right)-1$, then

$$
\overleftarrow{X}_{u}^{t}=\exp \left(\left(\sigma^{2}-\mu+\lambda\left(\alpha^{*}+\overleftarrow{\alpha}^{*}\right)-\lambda \overleftarrow{\alpha}^{*}-\frac{1}{2} \sigma^{2}\right) u-\sigma \overleftarrow{W}_{u}+\overleftarrow{L}_{u}\right)
$$

The process identified in (33) is a sub-martingale, if $\sigma^{2}-\mu+\lambda\left(\alpha^{*}+\overleftarrow{\alpha}^{*}\right) \geq 0$

which translates to condition (31). Further, the distribution of $\left(\overleftarrow{X}_{u}\right)$ as a stochastic process is time-consistent in $t$. Therefore, the result follows from Proposition 6 .

Note that Proposition 7 provides an alternative proof of Proposition 2, part 1 by setting $\lambda=0$, i.e., not permitting jumps. As indicated earlier, many other examples can be constructed from Lévy processes using a similar analysis as in the proof of Proposition 7, but involving more advanced methods from stochastic analysis.

\section{Conclusions}

We extended the class of peacock processes by restricting the class of functions for which an increase in the convex order is required, to increasing convex functions only. We showed that the resulting class of processes has relevance in many different contexts, and includes important examples of processes, which have been excluded from the peacock literature so far. Due to relationships with the case of an Australian options and the peacock frame- 
work, we named this new class of processes lyrebirds, in acknowledgment of the Australian bird, who shares many similarities to the Asian peacock. We expect that many of the results obtained in the peacock context, can be generalized to the lyrebird world. Our explicit results include Asian and Australian options in the Black-Scholes model with drift, the general arbitrage free case for the Asian variant and the Merton type jump diffusion case for the Australian variant, as well as the case of an Asian option on an exponential Brownian bridge, which is of relevance in the pricing of bond and currency options.

\section{Appendix}

Lemma A 1. Let $\left(W_{s, t}\right)$ be a standard Wiener sheet. Then for $\nu \in[0,1]$ and $0 \leq s \leq t$ the random variable $\left(W_{\nu, t}-\nu W_{1, t}\right)-\left(W_{\nu, s}-\nu W_{1, s}\right)$ is normal distributed with expectation zero and variance $\nu(1-\nu)(t-s)$.

Proof. Let $X:=\left(W_{\nu, t}-\nu W_{1, t}\right)-\left(W_{\nu, s}-\nu W_{1, s}\right)$ denote the expression under consideration. It follows from (41) and the fact that $W_{s, t}$ is Gaussian, that $X$ is normal distributed with expectation zero. The variance of $X$ can be computed as follows:

$$
\begin{aligned}
\operatorname{var}(X)= & \operatorname{var}\left(W_{\nu, t}\right)+\operatorname{var}\left(W_{\nu, s}\right)+\nu^{2} \operatorname{var}\left(W_{1, t}\right)+\nu^{2} \operatorname{var}\left(W_{1, s}\right) \\
& -2 \operatorname{cov}\left(W_{\nu, t}, W_{\nu, s}\right)-2 \nu \operatorname{cov}\left(W_{\nu, t}, W_{1, t}\right)+2 \nu \operatorname{cov}\left(W_{\nu, t}, W_{1, s}\right) \\
& +2 \nu \operatorname{cov}\left(W_{\nu, s}, W_{1, t}\right)-2 \nu \operatorname{cov}\left(W_{\nu, s}, W_{1, s}\right)-2 \nu^{2} \operatorname{cov}\left(W_{1, t}, W_{1, s}\right) .
\end{aligned}
$$

Using (41) and the assumption that $\nu \in[0,1]$, we obtain

$$
\begin{aligned}
\operatorname{var}(X) & =\nu t+\nu s+\nu^{2} t+\nu^{2} s-2 \nu s-2 \nu^{2} t+2 \nu^{2} s+2 \nu^{2} s-2 \nu^{2} s-2 \nu^{2} s \\
& =\nu(1-\nu)(t-s)
\end{aligned}
$$




\section{References}

[1] Ajevskis, V., Vitola, K. (2010) A Convergence Model of the Term Structure of Interest Rates. Review of Finance, Vol. 14, 727747

[2] Baker, D., Yor, M. (2009) A Brownian sheet martingale with the same marginals as the arithmetic average of geometric Brownian motion. Elect. J. Prob. 14(52), 1532-1540

[3] Ball, C.A., Torous, W. (1983) Bond Price Dynamics and Options. The Journal of Financial and Quantitative Analysis, Vol. 18, No. 4, 517-531

[4] Beiglböck, M., Juillet, N. (2016) On a problem of optimal transport under marginal martingale constraints. Annals of Probability (to appear)

[5] Bogso A.M. (2015) MRL order, log-concavity and an application to peacocks. Stochastic Processes and their Applications 125 (4), 1282-1306

[6] Bogso A.M. (2016) MRL order, weak decreasing stochastic order and lyrebird, working paper.

[7] Carr, P., Ewald, C.-O., Xiao, Y. (2008) On the Qualitative Effect of Volatility and Duration on Prices of Asian. Finance Research Letters, Volume 5, Issue 3, 162-171

[8] Chen, Jilong and Ewald, Christian-Oliver, On the Performance of the Comonotonicity Approach for Pricing Asian Option in Some Benchmark Models from Equities and Commodities (February 6, 2014). Available at SSRN: http://ssrn.com/abstract $=2391772$

[9] Cheng, S. (1991) On the Feasibility of Arbitrage-Based Option Pricing When Stochastic Bond Price Processes Are Involved. Journal of Economic Theory 53, 185-198 
[10] Davidson, R. (2010) Innis Lecture: Inference on Income Distributions. The Canadian Journal of Economics. Vol. 43, No. 4

[11] Delbaen, F., Shirakawa, H. (2002) A Note on Option Pricing for the Constant Elasticity of Variance Model. Asia-Pacific Financial Markets 2002, Volume 9, Issue 2, pp 85-99

[12] Doob, J.L. (1968) Generalized Sweeping-Out and Probability. Journal of Functional Analysis 2, 207-225.

[13] Ewald, C.-O., Menkens, O., Ting, S.H.M. (2013) Asian and Australian options: A common perspective. Journal of Economic Dynamics and Control, $37(5)$

[14] Ewald, C.-O., Yor, M. On Increasing risk, inequality and poverty measures: Peacocks, lyrebirds and exotic options. Journal of Economic Dynamics and Control, 59: 22-36 (2015).

[15] Handley, J.C. (2000). Variable purchase options. Review of Derivatives Research, 4(3):219-230, October 2000.

[16] Handley, J.C. (2003). An empirical test of the pricing of vpo contracts. Australian Journal of Management, 28(1):409-422, June 2003.

[17] Hirsch, F., Profeta, C., Roynette, B. and Yor, M. (2011) Peacocks and Associated Martingales with Explicit Constructions. Springer.

[18] Jeanblanc, M., Yor, M, Chesney, M. (2009) Mathematical methods for financial markets. Springer.

[19] Källblad, S., Tan, X. Touzi, N. (2015) Optimal Skorohod embedding given full marginals and Azema-Yor peacocks. Preprint at arXiv:1503.00500 [math.PR]

[20] Kellerer, H.G. (1972) Markov Komposition und eine Anwendung auf Martingale. Math. Ann. 198, 99-122 
[21] Merton, R. C. (1976) Option pricing when underlying stock returns are discontinuous. Journal of Financial Economics 3 125-144

[22] Revuz, D. and Yor, M. (2004) Continuous Martingales and Brownian Motion. Springer, Grundlehren der mathematischen Wissenschaften .

[23] Strassen, V. (1965) The existence of probability measures with given marginals. The Annals of Mathematical Statistics 36, 423-439.

[24] Yang, Z., Ewald, C.-O., Menkens, O. (2011) Pricing and hedging of Asian options: quasi-explicit solutions via Malliavin calculus. Mathematical Methods of Operations Research 74, 93-120 